\section{A Novel Superoxide Dismutase of High Molecular Weight from Bovine Liver STEFAN MARKLUND}

\section{Department of Chemistry, Section of Physiological Chemistry, University of Umea, S-901 87 Umea, Sweden}

Gince the discovery of the enzymic $N_{\text {activity in } 1969,{ }^{1} \text { superoxide dismutases }}$ have been isolated from a wide variety of organisms. Eukaryotes have been found to contain enzymes containing $2 \mathrm{Cu}$ and $2 \mathrm{Zn}$ whereas the enzymes from prokaryotes contain $2 \mathrm{Mn} .^{2}$ Both types of enzymes have molecular weights in the range $30000-$ 40 000. In mammals superoxide dismutases have been isolated from several tissues, e.g. liver, ${ }^{3}$ brain, ${ }^{3}$ heart, ${ }^{4}$ and all have been found to be identical to the enzyme found in erythrocytes: $:^{3,4}$ erythrocuprein.

When hemolysates are run on polyacrylamide gel electrophoresis and stained for superoxide dismutase activity one group of bands is found, apparently representing erythrocuprein. However, many tissue homogenates show two groups of bands (Fig. 1a), one fast, apparently

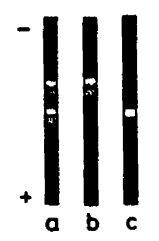

Fig. 1. Electrophoresis in polyacrylamide gels at $\mathrm{pH} 8.9$ with staining for superoxide dismutase activity. ${ }^{10}$ (a) Liver homogenate; (b) High molecular weight fraction from gel chromatography, cf. Fig. 2; (c) Low molecular weight fraction, $c f$. Fig. 2.

given by erythrocuprein and one slow, the origin of which is so far unknown. The two groups in bovine liver homogenates are easily separated by gel chromatography, the electrophoretically slow fraction apparently having the higher molecular weight.

The present communication reports some properties of the high molecular weight component, shown to have a molecular weight of $\approx 73000$ and an isoelectric point around $\mathrm{pH}$ 7.9. Some studies on metal content were also performed.
Materials and methods. Bovine liver was obtained fresh from a local slaughterhouse and stored at $-70^{\circ} \mathrm{C}$. It was cut into thin slices, thoroughly washed in icecold saline and homogenized in a Turmix with 3 volumes of $5 \mathrm{mM}$ $\mathrm{Na}$ phosphate, $\mathrm{pH}$ 7.4. After centrifugation of the homogenate at $30000 \mathrm{~g}$ for $60 \mathrm{~min}$, the supernatant was applied to a Sephadex G-200 column $(9.2 \times 70 \mathrm{~cm})$ and eluted with $30 \mathrm{mM}$ Na phosphate, pH 7.40 at $60 \mathrm{ml} \mathrm{h}^{-1}$.

Bovine erythrocuprein was prepared by the method of McCord and Fridovich ${ }^{5}$ as modified by Weser et al. ${ }^{6}$

The molecular weight was determined according to Andrews ${ }^{7}$ on a Sephadex G-200 (preswollen $>6$ months) column $(2.5 \times 70 \mathrm{~cm}$, elution rate $2 \mathrm{ml} \mathrm{cm} \mathrm{cm}^{-2} \mathrm{~h}^{-1}$ ) with $30 \mathrm{mM} \mathrm{Na}$ phosphate, $\mathrm{pH} 7.4+0.2 \mathrm{M} \mathrm{NaCl}$ as eluent. The column was calibrated with human gamma globulin, human transferrin, bovine serum albumin, and bovine myoglobin.

Discontinuous polyacrylamide gel electrophoresis was performed according to Maurer ${ }^{8}$ in $7.5 \%$ gels at $\mathrm{pH} 8.9$. Isoelectric focusing was performed for $72 \mathrm{~h}$ at $600 \mathrm{~V}$, in the $\mathrm{LKB}$ column $8101,110 \mathrm{ml}$ (LKB Stockholm, Sweden) with $1 \%$ Ampholine, pH 3-10, according to LKB's suggestions and with a sucrose density gradient. Metal analyses were per. formed by atomic absorption spectroscopy in a graphite furnace. ${ }^{9}$

Results and discussion. Fig. la shows a polyacrylamide gel electrophoresis of a bovine liver homogenate stained for superoxide dismutase activity. The activity is found to be divided into two groups of bands.

The dismutase activity of a liver homogenate is also separated into two fractions upon chromatography on Sephadex

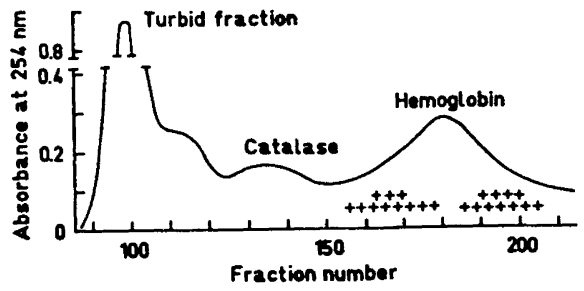

Fig. 2. Chromatography of bovine liver homogenate on Sephadex G-200. The preparation of the homogenate and the chromatography were carried out as described in Materials and Methods. Superoxide dismutase activity was detected by the nitro blue tetrazolium-photo. reduction method, ${ }^{10}++$.

Acta Chem. Scand. 27 (1973) No. 4 
G-200, Fig. 2. The high molecular weight component corresponds to the electrophoretically slow group and the low molecular weight component corresponds to the fast group, Fig. $1 b$, c. The position of the latter group in the chromatogram corresponds to a molecular weight $\approx 30000$ and it cannot be distinguished from bovine erythrocyte erythrocuprein on a poly acrylamide gel stained for superoxide dismutase. Hence it is probably the erythrocuprein present in liver (hepatocuprein). ${ }^{\mathbf{3}}$

The yield of the high molecular weight component varies from liver to liver. The activity in the liver used in the present investigation was high and about equal to that of the low molecular weight component. It has apparently not been described before and was subjected to a number of investigations.

All assay procedures for superoxide dismutase are indirect and there is therefore a risk that components lacking true superoxide dismutase activity may give rise to positive reactions. However, three different procedures for superoxide dismutase determination gave the same result for the high molecular weight fraction; the xanthine oxidase-cytochrome $\mathrm{C}$ procedure, ${ }^{5}$ the photoreduction of nitro blue tetrazolium, ${ }^{10}$ and the reduction of nitro blue tetrazolium by NADH mediated by phenazine methosulfate.11 Further, the fraction contained no cytochrome $\mathrm{C}$ oxidizing activity. However, the method employ. ing the autooxidation of adrenalin at $\mathrm{pH}$ $10.2^{12}$ could not be used for the high molecular weight fraction which, unlike erythrocuprein, apparently loses its activity at this $\mathrm{pH}$.

The activity was not affected by exposure to $10 \mathrm{mM}$ EDTA at $\mathrm{pH} 6.6$ at room temperature for a few hours. Neither did exposure to $4 \mathrm{M}$ urea for an hour affect its activity or electrophoretic mobility.

The apparent molecular weight as determined by chromatography on a calibrated Sephadex G-200 column was $=73000$. The molecular weight is hence much higher than those of previously described copper- or manganese-containing superoxide dismutases. Isoelectric focusing (Fig. 3) revealed an isoelectric point of $\mathrm{pH}$ 7.9 , much higher than that of bovine erythrocuprein, $4.95 .^{13}$ The zone of activity in the polyacrylamide gel (Fig. 1b) and the band in isofocusing (Fig. 3) are fairly broad. This is indicative of an inhomo. geneity in the high molecular weight fraction. Whether this is due to the presence of isoenzymes or to secondary changes in the protein cannot be decided at present.

The band of superoxide dismutase activity from the electrofocusing was analyzed for $\mathrm{Mn}$ and $\mathrm{Cu}$ content by means of atomic absorption in a graphite furnace. The enzyme activity correlated with the presence of $\mathrm{Mn}$ but the region also contained much $\mathrm{Cu}$. The active fraction is probably not pure. Some copper complexes display a superoxide dismutase activity approaching $\leq 7.5 \%$ of that of erythrocuprein. ${ }^{\mathbf{1 4}}$ Irrespective of whether the active component contains all $\mathrm{Cu}$ or Mn or both, the activity per metal in the

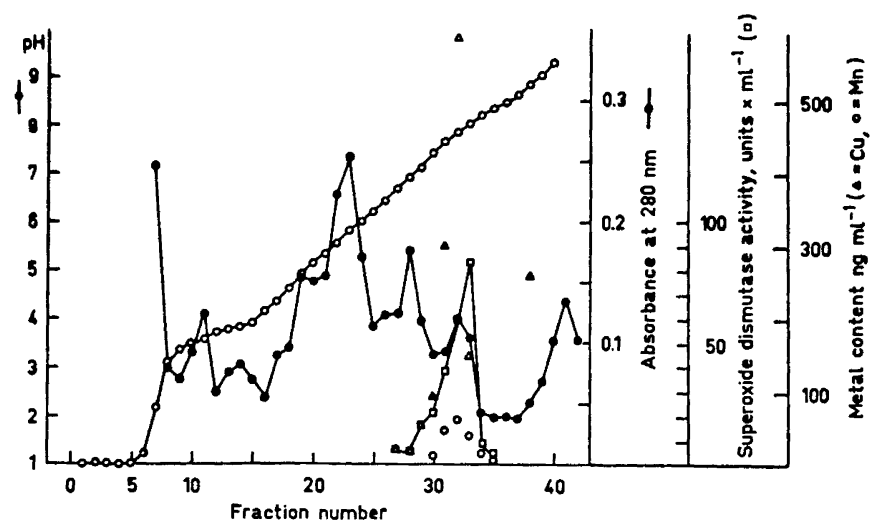

Fig. 3. Isoelectric focusing of the high molecular weight superoxide dismutase. Superoxide dismutase activity was determined according to McCord and Fridovich. ${ }^{5}$ Metals were de. termined by means of atomic absorption spectroscopy ${ }^{\circ}$.

Acta Chem. Scand. 27 (1973) No. 4 
most active fraction (33) is $\geq 50 \%$ of that of $\mathrm{Cu}$ in erythrocuprein. ${ }^{5}$ This, together with its resistance towards EDTA, seems to exclude that the activity is of a nonspecific metal-complex type.

Some people have genetic variant forms of erythrocuprein. ${ }^{15}$ Variants are not found in the electrophoretically slow component from placentas of such persons, which indicates different genetic control. ${ }^{16}$ Preliminary investigations indicate that an electrophoretically slow component staining for superoxide dismutase activity from $\mathrm{Kb}$ cells is associated with a particulate fraction, probably mitochondria, whereas a fast component is cytoplasmatic. ${ }^{16}$ The slow component may be related to the manganese containing superoxide dismutase recently mentioned as present in chicken liver mitochondria. ${ }^{17}$

Acknowledgement. I want to thank Dr. G. Lundgren for performing the atomic absorption spectroscopy.

1. McCord, J. M. and Fridovich, I. Fed. Proc. 28 (1969) 346.

2. Fridovich, I. Accounts Chem. Res. 5 (1972) 321.

3. Carrico, R. J. and Deutsch, H. F. J. Biol. Chem. 244 (1969) 6087.

4. Keele, B. B., McCord, J. M. and Fridovich, I. J. Biol. Chem. 246 (1971) 2875.

5. McCord, J. and Fridovich, I. J. Biol. Chem. 244 (1969) 6049.

6. Weser, U., Bunnenberg, E., Cammack, R., Djerassi, C., Flohé, L., Thomas, G. and Volter, W. Biochim. Biophys. Acta 243 (1971) 203

7. Andrews, P. Methods Biochem. Anal. 18 (1970) 1 .

8. Maurer, H. R. Disk-Electrophorese, Walter de Gruyter, Berlin 1968.

9. Lundgren, G. and Johansson, G. Talanta. Submitted.

10. Beauchamp, C. and Fridovich, I. Anal. Biochem. 44 (1971) 276.

11. Nishikimi, M., Rao, N. A. and Yagi, K. Biochem. Biophys. Res. Commun. 46 (1972) 849.

12. Misra, H. P. and Fridovich, I. J. Biol. Chem. 247 (1972) 3170.

13. Bannister, J., Bannister, W. and Wood, E. Eur. J. Biochem. 18 (1971) 178.

14. Joester, K.-E., Jung, G., Weber, U. and Weser, U. FEBS Lett. 25 (1972) 25.

15. Beckman, G. Hereditas. In press 1973.

16. Beckman, G., Lundgren, E. and Tärnvik, A. In manuscript.

17. Fridovich, I. Biochem. Soc. Trans. 1 (1973) 48.

Received March 22, 1973.

\section{Ring Inversion in Substituted Spiro[2.5] octanes \\ PER KOLSAKER, HANS JOHAN \\ STORESUND, JAN SCHAUG and GRETE WØIEN LARSEN}

Department of Chemistry, University of Oslo, Blindern-Oslo 3, Norway

Some of the interest in the field of inNersion of cyclohexane rings has been focused on compounds having hybridization different from $s p^{3}$ at one or more of the ring atoms. NMR spectroscopy has proven useful to evaluate rate constants for such processes provided the free energy of activation is larger than about $6 \mathrm{kcal} / \mathrm{mol}$. By this technique 1,4-dimethylenecyclohexane (I) has been studied and a $\Delta G^{\neq}$value of $7.5 \mathrm{kcal} / \mathrm{mol}$ was calculated for the inversion for which arguments are presented showing that this most likely is a chair-chair interconversion. ${ }^{1} \mathrm{~A}$ change in the hybridization from $s p^{2}$ to $s p^{3}$ as in 1,1,4,4-tetramethylcyclohexane (II) raises the barrier to inversion to $11.6 \mathrm{kcal} / \mathrm{mol} .^{2}$ An intermediate value of $10.7 \mathrm{kcal} / \mathrm{mol}$ has recently been found in dispiro[2.2.2.2] decane (III) and fits qualitatively in the picture of the ring atoms involved in substitution having a slightly higher $s$-character than in II. Methylenecyclohexane (IV) has also been found to exist predominantly in a chair
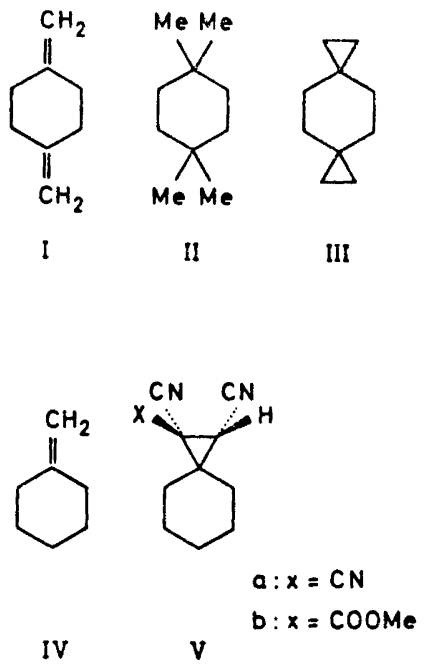

Acta Chem. Scand. 27 (1973) No. 4 